\title{
Transmission d'entreprises PME saines en Scop au regard de la relève de la direction : une étude exploratoire de faisabilité en France
}

\section{Turning healthy SMEs intoworker cooperatives and the issueof the change in management:An exploratory feasibility study in France}

\section{France Huntzinger et Thierry Jolivet}

\section{Numéro 316, mai 2010}

URI : https://id.erudit.org/iderudit/1020895ar

DOI : https://doi.org/10.7202/1020895ar

Aller au sommaire du numéro

Éditeur(s)

Association Recma

ISSN

1626-1682 (imprimé)

2261-2599 (numérique)

Découvrir la revue

\section{Citer cet article}

Huntzinger, F. \& Jolivet, T. (2010). Transmission d'entreprises PME saines en Scop au regard de la relève de la direction : une étude exploratoire de faisabilité en France. Revue internationale de l'économie sociale, (316), 58-71. https://doi.org/10.7202/1020895ar
Résumé de l'article

Partant des recherches sur les transmissions d'entreprises familiales, nous nous interrogeons sur la pertinence de leurs conclusions dans le cas du passage d'une PME saine en société coopérative de production (Scop). L'accent est mis sur l'aspect humain du processus, qui implique autant le prédécesseur, ou cédant, et le futur dirigeant que les salariés. Nous appuyant sur les résultats d'une enquête auprès de dirigeants de coopérative concernés par leur propre relève et acteurs dans des processus de transmission en Scop, nous montrons qu'il faut nuancer l'importance des éléments explicatifs de la transition - place du deuil, rôle des étapes clés, rôle des salariés -, l’intégration du nouveau comme le désengagement de l'ancien étant facilités par rapport aux cas d'entreprises familiales classiques. A partir de l'expérience des successions au sein même des Scop, cette étude exploratoire a permis d'identifier des conditions de transformation d'une PME saine en Scop dans un contexte où les acteurs du mouvement coopératif se sont plus fortement mobilisés que par le passé pour ce mode de création. 


\section{TRANSMISSION D'ENTREPRISES PME SAINES EN SCOP AU REGARD DE LA RELĖVE DE LA DIRECTION: UNE ÉTUDE EXPLORATOIRE DE FAISABILITÉ EN FRANCE}

\footnotetext{
* Maître de conférences hors classe et professeur des universités en sciences de gestion, université du Maine-Laboratoire Gains-Argumans. Mél.: France. Huntzinger@univ-lemans.fr ; Thierry.Jolivet@univ-lemans.fr. * Cet article est une version adaptée de la communication présentée à la lre Conférence mondiale de la recherche en économie sociale, organisée à Victoria (Colombie-Britannique, Canada) du 22 au 25 octobre 2007, et de sa publication en espagnol dans la revue Ciriec España ( ${ }^{\circ}$ 66/2009, p. 129-146); sommaire consultable sur Recma.org.
}

par France Huntzinger et Thierry Jolivet*

Partant des recherches sur les transmissions d'entreprises familiales, nous nous interrogeons sur la pertinence de leurs conclusions dans le cas du passage d'une PME saine en société coopérative de production (Scop). L'accent est mis sur l'aspect humain du processus, qui implique autant le prédécesseur, ou cédant, et le futur dirigeant que les salariés. Nous appuyant sur les résultats d'une enquête auprès de dirigeants de coopérative concernés par leur propre relève et acteurs dans des processus de transmission en Scop, nous montrons qu'il faut nuancer l'importance des éléments explicatifs de la transition - place du deuil, rôle des étapes clés, rôle des salariés -, l'intégration du nouveau comme le désengagement de l'ancien étant facilités par rapport aux cas d'entreprises familiales classiques. A partir de l'expérience des successions au sein même des Scop, cette étude exploratoire a permis d'identifier des conditions de transformation d'une PME saine en Scop dans un contexte où les acteurs du mouvement coopératif se sont plus fortement mobilisés que par le passé pour ce mode de création**.
(1) Avec l'appui de la Confédération générale des Scop (CGScop) et de son Observatoire inter-universitaire sur le management et la gestion des Scop (Obgescop), dans le cadre de conventions d'étude avec l'université du Maine. (2) Participer (revue éditée par la CGScop et diffusée au sien du réseau des Scop), $n^{\circ} 609$, févriermars 2005, p. 14-18.
$\mathrm{N}$ otre recherche ${ }^{(1)}$ sur le management des coopératives de production (Bataille-Chédotel et Huntzinger, 2004) nous conduit aujourd'hui à nous interroger sur la stratégie des acteurs du mouvement Scop (congrès de Nantes en 2004, de Poitiers en 2008) qui se sont mobilisés sur les opportunités de proposer la transmission aux salariés de certaines entreprises familiales, artisanales et PME saines sous forme coopérative $^{(2)}$, en liaison avec les prescripteurs classiques qui reconnaissent peu à peu la formule Scop comme une solution avantageuse sur le plan économique comme sur le plan humain. En effet, la question des successions se pose principalement en raison de l'allure de la pyramide des âges: nombre de patrons proches du départ à la retraite n'ont pas trouvé de solution. Mais ce phénomène est aussi présent dans le secteur des Scop, où les fondateurs de Scop créées dans les années 70 et 80 arrivent à l'âge 
(3) Un potentiel de plus de 1000 PME par an sur dix ans (Participer, $n^{\circ} 610$, avril-mai 2005, p. 14-22).

(4) Ce terme, associé aux mots "PME " ou " entreprise ", fait référence au statut juridique de droit commun distinct du statut coopératif.

(5) Transformation d'une entreprise saine en Scop, selon la terminologie interne à la CGScop. de la retraite ${ }^{(3)}$ et doivent passer le relais de la direction. Or, le passage d'une PME en Scop comme la succession du dirigeant dans une Scop renvoient à l'existence d'acteurs clés: prédécesseur - cédant -, salariés coopérateurs ou futurs coopérateurs, à la fois sensibles aux valeurs coopératives et capables d'une véritable démarche entrepreneuriale.

Parmi les travaux portant sur les transmissions d'entreprise, certainsauteurs mettent l'accent sur la nécessaire prise en compte de "l'environnement psychologique " du cédant (Geye et Bah, 2003) ou du processus de désengagement du prédécesseur (Cadieux, 2005; Fattoum et Fayolle, 2002), ou encore des "freins psychologiques" (Pailot, 1998) et de socialisation du repreneur (Boussaguet, 2003). Cette importance accordée au facteur humain dans la compréhension de la réussite ou de l'échec des transmissions d'entreprise nous semble pertinente et bienvenue dans le cas des Scop. Les nombreuses études sur l'entreprise familiale, notamment, contrastent avec l'aspect embryonnaire des recherches portant sur les Scop. Notre démarche est donc originale par son caractère exploratoire pour comprendre les conditions des transformations de PME saines en Scop, qui cumulent les problèmes de changement de dirigeant et de mobilisation d'un nouveau sociétariat avec les salariés de la PME. Pour cela, nous avons émis l'hypothèse que l'expérience des dirigeants de Scop qui doivent organiser leur succession à la direction en interne puisse fournir un éclairage pertinent pour répondre aux questionnements concernant la transformation sur le plan humain d'une entreprise "classique ${ }^{(4)}$ " en Scop: la forme coopérative ne peut-elle pas offrir aux dirigeants de PME une manière de réduire ce " coût psychologique » et apporter des réponses possibles au problème du désengagement du dirigeant? La bonne gouvernance du processus ne suppose-t-elle pas aussi l'engagement des acteurs représentatifs du mouvement Scop, dont la pratique dite des mutations ${ }^{(5)}$ est une tradition qui mérite d'être soulignée?

Dans cet article, nous proposons en premier lieu d'analyser les apports des travaux sur les entreprises classiques qui privilégient le facteur humain, puis de montrer la place historique des mutations dans les créations de Scop justifiant le rôle des acteurs dirigeants du mouvement dans ces procédures. Enfin, nous tâcherons, à partir d'une enquête exploratoire par entretiens approfondis auprès de quatre dirigeants de Scop, eux-mêmes acteurs à la fois impliqués dans un processus de succession en interne et dans l'accompagnement de patrons de PME en quête de relève, de dégager les spécificités de la forme coopérative en cas de changement de direction et d'en faire émerger quelques pistes sur les conditions de faisabilité d'une transformation de PME saine en Scop.

\section{Les aspects humains du changement de direction}

Les recherches évoquent la théorie du deuil comme mécanisme explicatif central de la difficulté ressentie par le cédant. La perte d'un lien 
(6) Définie comme la "présence simultanée, dans la relation à un même objet, de tendances, d'attitudes et de sentiments opposés ", selon Laplanche et Portalis (1998), cité par Gueye et Bah (2003, p. 1363). d'attachement fort est vécue comme un traumatisme et viendrait freiner l'ardeur du cédant à transmettre et compromettre la réussite du processus de transmission. Gueye et Bah (2003), par exemple, soulignent que ce deuil est double, puisqu'il concerne l'entreprise comme objet d'attachement, mais également l'apport symbolique que la possession de cet objet procure: statut et image sociale. Fattoum et Fayolle (2002) citent ainsi plusieurs auteurs qui soulignent la perte d'identité et de pouvoir, le sentiment d'inutilité, car se désengager de son entreprise revient à renoncer à une partie de soi-même, voire conduit à une « mort symbolique ». Pailot (1998) propose en outre une analyse du lien dirigeant-firme à partir du concept d'emprise emprunté à l'épistémologie dialectique. Le cas qu'il étudie lui permet de rendre compte de "l'attachement viscéral de certains dirigeants pour leur entreprise ", expliquant alors la difficulté ressentie à "faire le deuil» lors du passage de relais.

\section{Ambivalence et confiance}

Ces travaux distinguent pour la plupart le transfert de propriété du transfert de direction. Pour notre part, nous nous focaliserons sur le second aspect, même si nous évoquerons aussi le transfert de propriété compte tenu de la spécificité de la transformation de PME classiques en Scop, qui suppose de transformer des salariés en sociétaires et donc en copropriétaires, voire en co-entrepreneurs. Ce transfert de direction est décrit comme un processus en quatre étapes: 1) l'incubation, initiation du successeur pressenti ; 2) le choix, la préparation et l'intégration du successeur; 3) le règne conjoint, entrée en fonction officielle du successeur et phase de transmission des connaissances et du pouvoir ; 4) le désengagement du prédécesseur. Ces quatre étapes ont été inégalement étudiées: les travaux portent principalement sur la quatrième, le désengagement du prédécesseur. Cadieux (2005) fait ainsi référence à la littérature sur la retraite et la transition de rôle et propose un modèle de réussite fondé sur l'existence de rites de séparation et de rites d'incorporation permettant de mieux vivre les périodes critiques tant pour le cédant que pour le repreneur. L'étape du règne conjoint fait toutefois l'objet d'un développement particulier chez Gueye et Bah (2003) et chez Fattoum et Fayolle (2002). Les premiers mobilisent le concept d'origine psychanalytique d'ambivalence ${ }^{(6)}$ pour expliquer que le règne conjoint peut être marqué par l'indécision du cédant qui oscille entre deux attitudes contradictoires: passer le relais et garder le contrôle. Cette ambivalence va venir entraver le nécessaire transfert de connaissances du cédant vers le successeur, gage de réussite du processus de transmission. Les connaissances tacites, notamment, ne seront transférées que si une relation de confiance s'établit entre les deux protagonistes. Celle-ci suppose du temps, comme le notent Reynaud et Richebé (2007, p. 7, note 8): "La confiance n'est pas déjà là, elle se construit progressivement et elle est un résultat du développement même de la relation d'échange. " La réussite du règne conjoint repose donc sur l'apparition et le développement de la confiance. 
(7) Selon le modèle de Le BretonMiller et Miller (2004).
Par ailleurs, Fattoum et Fayolle (2002) insistent eux aussi sur la nécessité de la confiance, mais ils développent en outre une modélisation de l'ensemble de la relation prédécesseur-successeur en avançant que celle-ci est caractérisée par trois états: une relation tendue dans la phase d'intégration du successeur; une relation complice au cours du règne conjoint; une relation de nouveau tendue lors du désengagement du cédant.

\section{Socialisation du repreneur et planification de la transmission}

Un autre aspect du processus de transmission est celui du rôle des salariés dans la socialisation du repreneur (Boussaguet, 2003, p. 410-417). S'appuyant sur le concept de socialisation organisationnelle et sur la littérature qui lui est consacrée, S. Boussaguet distingue trois phases dans le processus:

- la socialisation anticipée, qui comprend la formulation des attentes avant l'entrée dans l'entreprise et les "mises en auvre par l'organisation d'interventions destinées à faciliter l'intégration [...]";

- la socialisation active, qui débute avec la prise de fonction. Les attentes sont confrontées à la réalité (reality choc), elles sont confirmées ou infirmées; - l'intégration, qui constitue la dernière phase du processus. C'est la fin du règne conjoint, le retrait du cédant est effectif. En outre, le repreneur "s'affirme dans son nouveau rôle organisationnel et [...] est reconnu légitime dans le rôle qu'il exerce".

Enfin, certains travaux s'attachent à décrire plutôt les activités de planification du processus de transmission. Saint-Cyr et Richer (2005), notamment, constatent dans leur enquête que celles relatives à l'établissement des critères de choix du successeur et à la communication de ces critères aux intéressés, ainsi que celles relatives à sa préparation sont assez conformes aux "meilleures pratiques » en la matière ${ }^{(7)}$. En revanche, elles soulignent que le désengagement du prédécesseur apparaît moins bien planifié dans les entreprises de plus petite taille ou dans celles qui expérimentent le transfert générationnel pour la première fois. Cette dernière approche identifie certes les aspects planifiables du processus, mais n'intègre pas la dimension psychologique du vécu des protagonistes.

\section{La mutation en Scop: une tradition centenaire du mouvement coopératif méconnue face à un enjeu sociétal}

(8) Organisés à l'université de Limoges en 2004 et 2005 et à celle du Mans en 2006.
L'importance sociétale de la question de la relève des dirigeants d'entreprise nous a conduits à procéder à une analyse documentaire basée sur des sources statistiques internes à la CGScop, ainsi que sur des propos relatés dans les revues professionnelles du mouvement coopératif ou à l'occasion de " carrefours ${ }^{(8)}$ " où participaient à la fois des universitaires et des coopérateurs. Le mouvement coopératif de production peut ainsi se prévaloir de son expérience en matière de transformation pour devenir un des intervenants potentiels sur le « marché » de la transmission de PME saine. 
(9) Yves Fouquet, président de la commission transmission au Conseil supérieur de l'ordre des experts-comptables, in Participer, $n^{\circ} 610$, ibid.

(10) Réanimation (transformation en Scop d'une entreprise en difficulté), création ex nihilo, recréation (création d'une Scop par tout ou partie des salariés d'une société liquidée).

(11) Coordinateur Patrick Lenancker, vice-président de la CGScop et élu président en 2006, succédant à Patrick Ségouin

(12) Participer, n 610, p. 19.

(13) François Kerfourn, directeur de I'union régionale des Scop Ouest, III Carrefour universitaire du management Scop, "La gouvernance des Scop en question? Regards croisés ", université du Mans, Argumans-Gains, 5 avril 2006.

(14) Fichier CGScop.
(15) Au sens de la pérennité du projet, pour reprendre la distinction proposée par Mignone cité par Barbot et Richomme-Huet (2004, p. 3).

(16) Nous utilisons la classification, en nombre de salariés, proposée par Mahé de Boislandelle (1998): TPE, très petite entreprise (1 à 9); $P E$, petite (10 à 49); $M E$, moyenne (50 à 199); $M G E$ moyenne ou grande (200 à 500).

\section{L'enjeu sociétal}

Un rapport du Conseil économique et social de 2004 mettait en évidence l'incertitude sur l'avenir de près de 700000 PME et TPE en France dont les dirigeants seraient amenés à partir à la retraite entre 2005 et 2015. Pour les principales institutions professionnelles et territoriales qui se mobilisent, la transmission en Scop est le plus souvent la solution ultime pour assurer la pérennité de l'entreprise classique saine sans successeur ou repreneur, avec l'appui des acteurs du mouvement coopératif. Elle devient depuis peu une solution reconnue parmi d'autres comme le "moyen à la fois le plus serein et le plus sécurisant de transmettre son entreprise (9)".

Dans quelle mesure le mouvement Scop s'intéresse-t-il à cette modalité de création comparativement aux autres origines ${ }^{(10)}$ ? Existe-t-il un profil type de PME qui conduirait au succès de cette transformation? Combien d'entreprises sont susceptibles d'être transformées en Scop? L'ambitieux plan d'action publié dans un livre blanc ${ }^{(11)}$ suite au Congrès national des coopérateurs en 2004 a suscité des études de marché dans les unions régionales, notamment en Rhône-Alpes, où a été identifiée une cible potentielle de 4000 PME de trois à cinquante salariés à transformer en Scop dans les dix ans à venir, dans l'industrie et les services aux entreprises demandant du personnel qualifié, installées en zone rurale ou périurbaine et bénéficiant souvent d'une activité de niche ${ }^{(12)}$. Ces chiffres sont à nuancer par les études de faisabilité au cas par cas: "Souvent les dirigeants à partir de 55 ans se mettent en roue libre et font perdre de la valeur à leur entreprise. Le tiers des entreprises ne serait pas reprenable ${ }^{(13)}$. "

\section{Une tradition centenaire mais marginale dans I'histoire du mouvement Scop}

Depuis 1907, on a dénombré 523 transformations d'entreprises saines en Scop, dont 233 sont encore vivantes en juin $2007^{(14)}$. Le taux de survie à cinq ans est de $84,5 \%$ et à dix ans de $79 \%$, alors que d'après l'Insee ce taux s'établit à $51 \%$ pour l'ensemble des entreprises. Cependant, ce mode de création représente en moyenne à peine $10 \%$ des Scop créées et un tiers de l'ensemble des reprises en Scop, les deux autres tiers étant des réanimations ou des recréations, qui affichent également un taux de survie nettement supérieur à la moyenne (71\%). Il est donc manifeste que ce mode de transmission donne l'assurance d'une pérennité de l'entreprise ${ }^{(15)}$. Ces bons résultats sont à mettre notamment au crédit du rôle du réseau Scop dans l'étude des dossiers en amont comme dans l'accompagnement de proximité au démarrage.

\section{Le profil spécifique des transmissions volontaires en Scop}

Deux critères au moins sont à retenir: les secteurs d'activité et la taille. Le tableau 1 (en page suivante) montre que, sur un total de 467 Scop renseignées, 197 sont des TPE (42\%), 209 des PE (45\%), 51 des ME (11 \%) et 10 des MGE $(2 \%)^{(16)}$. Sil'on se réfère aux chiffres des transmissions durant les dix dernières années, sur 97 cas renseignés, $59 \%$ sont des TPE, $40 \%$ des PE 
et $1 \%$ uneME. La tendance est doncàla reprise d'entreprises de petite taille. La répartition de ces transmissions par secteurs d'activité (tableau 2, en page suivante) suit un profil quasiment similaire à celui de l'ensemble des Scop à ce jour, à l'exception du secteur "Fonderie, travail des métaux ", plus capitalistique et de taille moyenne plus élevée en général. Le domaine des services intellectuels et culturels (cabinets-conseils, bureaux d'études, services d'ingénierie...), qui s'est particulièrement développé depuis vingt ans, regroupe des entreprises de très petite taille qui sont composées de salariés souvent bien disposés vis-à-vis de la formule coopérative en raison notamment de la nature de leur métier, qui favorise le travail en équipe et la collégialité des décisions sur une base égalitaire. Cette distribution quasi parallèle renforce l'idée selon laquelle la transmission de PME en Scop est fortement initiée par les acteurs en place du mouvement coopératif, chaque dirigeant de Scop agissant dans son secteur auprès des prescripteurs traditionnels en la matière ou au sein même de leur groupement professionnel, en fonction de son savoir-faire et de ses relations, tant pour prospecter les entreprises que pour accompagner les éventuels cédants vers la solution Scop. La création de Scop par mutation est donc bien ancrée dans les pratiques du mouvement, sans avoir été cependant une orientation délibérée. Elle apparaît dans les axes stratégiques du congrès de Poitiers en 2008 et mérite une attention particulière sur les nouvelles méthodes d'approche et les nouveaux dispositifs de portage coopératif.

\section{La spécificité de la relève de la direction en Scop? Succession interne et transmission en Scop: regards croisés}

\section{Méthodologie de recherche}

Notre problématique de recherche nous a conduits à privilégier une démarche exploratoire qui permet d'accéder à une première étape de compréhension d'un phénomène que l'on ne peut mesurer. Il convenait d'utiliser une méthodologie qualitative (Wacheux, 1996) essentiellement fondée sur des entretiens non directifs, intégralement retranscrits, réalisés

\section{Tableau 1}

Répartition des mutations par taille lors de la création

\begin{tabular}{|r|r|r|r|r|r|r|}
\hline $1-4$ salariés & $\begin{array}{r}5-9 \text { salariés } \\
\text { TPE }\end{array}$ & $\begin{array}{r}10-30 \\
\text { salariés PE }\end{array}$ & $\begin{array}{r}31-49 \\
\text { salariés PE }\end{array}$ & $\begin{array}{r}50-100 \\
\text { salariés ME }\end{array}$ & $\begin{array}{r}101-199 \\
\text { salariés ME }\end{array}$ & $\begin{array}{r}200-500 \\
\text { salariés MGE }\end{array}$ \\
\hline 77 & 120 & 160 & 49 & 33 & 18 & 10 \\
\hline
\end{tabular}

Source: fichier CGScop. 


\section{Tableau 2 \\ Répartition des mutations par activités}

\begin{tabular}{|l|r|r|r|}
\hline $\begin{array}{l}\text { Secteur d'activité } \\
\text { (selon la classification CGScop) }\end{array}$ & Nombre & \% & \% ensemble Scop 2006 \\
\hline Bâtiment, travaux publics et activités connexes & 165 & 31,5 & 32 \\
\hline Industrie du livre & 35 & 6,7 & 7 \\
\hline Fonderie, travail des métaux & 39 & 7,5 & 11 \\
\hline Bois, ameublement, carton, verre, céramique & 14 & 2,7 & 3 \\
\hline Alimentation, agriculture, horticulture & 18 & 3,5 & 4 \\
\hline Cuirs et étoffes & 18 & 3,5 & 2 \\
\hline Prestations intellectuelles et culturelles & 136 & 26 & 25 \\
\hline Prestations de services matériels & 71 & 13,6 & 14 \\
\hline Divers & 27 & 5 & 2 \\
\hline Total & 523 & 100 & 100 \\
\hline
\end{tabular}

Source: fichier CGScop.

(17) Le "montagnard" est un dirigeant qui a gravi les échelons hiérarchiques au sein de la Scop jusqu'à devenir le PDG et qui a obtenu plusieurs renouvellements de son mandat. II a tendance à diriger en étroite collaboration avec son conseil d'administration pour une gouvernance collective. L'« héliporté " est un dirigeant en provenance d'une entreprise classique qui est entré dans la Scop à un niveau élevé de la hiérarchie et a assumé la fonction de PDG rapidement après son arrivée. II a tendance à diriger avec des comités et sollicite le CA plutôt comme " aiguillon " et dans une relation de confiance. en 2006 et 2007, comprenant en partie des récits de vie, d'une durée de trois à quatre heures, auprès de quatre dirigeants de Scop encore en poste, mais susceptibles de passer le relais dans les cinq ans à venir et de surcroît reconnus comme acteurs responsables ou "militants" au sein du mouvement sur le terrain des transmissions de PME en Scop. Il s'agissait non pas de procéder à des études de cas, mais de faire émerger un savoir concret et des points de vue issus du terrain, afin de les formaliser en vue d'une compréhension initiale d'une opération délicate sur le plan humain (la succession interne et a fortiori la transformation de PME en Scop). Ils ont été choisis en accord avec des responsables du réseau CGScop comme personnes ressources majeures pour leur réflexion et leur expérience personnelles sur leur propre succession à venir et sur la stratégie de transformation de PME saines en Scop compte tenu du contexte actuel dans le tissu économique des PME sans successeur. Ils correspondent à des profils de dirigeants identifiés lors d'une précédente enquête sur la typologie des modes de gouvernance coopératives: deux " montagnards " et deux "héliportés» (Bataille-Chédotel, Huntzinger, 2004) ${ }^{(17)}$. Deux d'entre eux faisaient déjà partie de l'échantillon des dix PDG interrogés; les deux autres correspondent respectivement à chacun des deux profils précédents. Partant de notre hypothèse de travail, nous présenterons les résultats de notre exploration en nous appuyant sur les entretiens où furent abordées, d'une part, la problématique de la succession interne dans la Scop et, d'autre part, celle des transformations en Scop de PME saines auxquelles les responsables interrogés ont participé. Les réponses obtenues sur 
(18) Rappelons que, selon les statuts légaux en France, conformément aux principes coopératifs, ces parts sociales sont remboursées au taux nominal éventuellement augmenté de celui de l'inflation, sachant qu'un sociétaire ne peut jamais détenir plus du quart du capital social de la coopérative. Ceci est la conséquence directe du principe des réserves impartageables.
(19) Cadieux (2005, p. 43) observe deux types de rôles: ceux auprès de l'organisation (administrateur, symbole, observateur, consultant...) et ceux auprès du successeur (facilitateur ou confident). Dans le cas des Scop, on peut penser que le premier rôle sera mieux accepté et plus pertinent que le second.

(20) Au sens de Bataille-Chédotel et Huntzinger (2004). le premier thème confrontées au second sous le regard des études déjà existantes sur les successions dans les entreprises familiales et artisanales nous ont permis de préciser la spécificité en la matière de la forme d'entreprise Scop et de dégager quelques enseignements sur les conditions de réussite de la transformation d'une PME saine en Scop comme solution au problème de la relève du patron.

\section{Les spécificités de la forme Scop au regard des processus de changement de dirigeant}

Nous allons maintenant examiner les résultats et conclusions présentés dans la première partie à la lumière de nos entretiens pour tenter de dégager les spécificités du processus de transmission de la direction d'entreprise dans le cas d'une Scop.

\section{La place du deuil}

Concernant le phénomène de deuil comme élément explicatif central de la difficulté de désengagement du cédant, il faut distinguer la succession interne dans une Scop, où il n'y a pas de transfert de propriété de l'entreprise à l'exception du remboursement des parts sociales du "sortant " ${ }^{(18)}$, de la transformation d'une PME en Scop, qui implique un rachat de l'entreprise tout entière. Dans le premier cas, le deuil est atténué, car le cédant est préparé depuis sa prise de poste à passer le relais un jour, comme en témoigne un de nos interlocuteurs, PDG depuis treize ans et préparant actuellement sa succession: "Je suis l'entreprise, ça ne me met pas en péril, je vais sortir dans cinq ans [...], l'entreprise n'est qu'un passage. " Dans le second cas, la difficulté à faire le deuil se manifeste-telle pleinement comme dans le cas d'une entreprise classique? En réalité, un facteur vient là encore l'atténuer du fait que les nouveaux propriétaires, les salariés en place, sont connus du cédant et parfois même avec des liens de type affectif: "Le premier souci du dirigeant, c'est de voir sa boîte se pérenniser, que les salariés gardent leur emploi, [d'autant qu'ils] font partie de la "famille"."

De surcroit, dans les deux cas l'ancien dirigeant peut être amené à jouer un nouveau rôle dans la Scop, sur un poste du type chargé du développement par exemple, ce qui lui permet de réorganiser sa vie sans rupture radicale ${ }^{(19)}$. Le coût psychologique peut donc être diminué dans le cas des Scop par rapport aux transmissions d'entreprises classiques, mais ce n'est pas systématique. En effet, si le dirigeant était un « montagnard local ${ }^{(20)}$ », c'est-à-dire un fondateur qui a fait toute sa carrière dans l'organisation, on risque de retrouver les mêmes phénomènes d'« attachement viscéral » et de difficulté à « faire le deuil », y compris lorsque le management avait un caractère très collectif, car le départ engendre non seulement une perte de pouvoir, mais aussi une perte d'identité et de sentiment d'appartenance en raison de la rupture avec le groupe de coopérateurs: «La reconnaissance de sa contribution à la réussite de l'entreprise nest donc pas "valorisée" et son engagement n'est pas d'une certaine manière "solde" par la compensation 
(21) Modèle de gouvernance fréquemment choisi dans les Scop de taille moyenne, selon l'étude Bataille-Chédotel et Huntzinger (2004). financière et le "chèque" reçu. [...] Seule la reconnaissance symbolique du collectif des sociétaires, de ses pairs dans le mouvement Scop et éventuellement du successeur peut venir "combler cette dette" "(Gouil, 2006, p. 3). Ainsi, le lâcher-prise peut être douloureux.

\section{L'importance respective des quatre étapes}

Concernant la distinction des quatre étapes de transmission, cette grille de lecture appliquée aux Scop fait ressortir l'importance cruciale des deux premières alors que les études menées auprès des entreprises classiques s'attachent au rôle clé des deux dernières.

Tout d'abord, plus en amont, il s'agit pour le dirigeant de commencer à penser à sa sortie. Dans le cas spécifique d'une direction à deux têtes ${ }^{(21)}$, le dirigeant chargé du développement créatif de l'activité, âgé de 57 ans, se disait prêt à quitter la Scop pour terminer sa carrière ailleurs. En réalité, il est demeuré dans l'indécision: "Je ne peux pas partir tout de suite, parce que vous allez être dans la ... " Selon l'autre co-directeur, "il se juge irremplaçable " parce que l'incertitude règne encore sur son successeur: "Un potentiel en interne [n'est] pas exclu, [...] mais la situation n'est pas claire. " Ainsi, engager le processus trop tôt avec des personnes ambivalentes peut s'avérer perturbateur pour les membres de la coopérative: "Ça nous a "paumés" quasiment, parce que [...] il y en avait un qui était prêt à partir tout de suite."

Quand on commence à rechercher activement un leader - "Ne pas attendre d'aller chercher en externe ce que l'on peut construire en interne "-, le mode participatif peut être utilisé: "Je souhaitais avoir un successeur en place dans cinq ans, au plus tard en 2009. J'ai fait travailler mes cadres de direction, on a pris un bon sujet: la stratégie, ce qui permettait de voir un petit peu où ils voulaient aller et comment, quelles étaient leurs ambitions. Et à l'issue de ce travail j'ai été amené à constater avec les uns et les autres que nous n'avions pas le bon successeur à l'intérieur de la coopérative. " Le schéma classique est cependant de promouvoir un cadre au poste de directeur général dont le PDG actuel fera son successeur, mais ce scénario n'est pas forcément celui qui se réalisera: "Ça peut être quelqu'un qui a des valeurs, qui est dans un autre poste ailleurs et qui par son comportement, son dynamisme, son ouverture, par un tas de choses, va être en meilleure situation que celui que l'on a mis en poste de second. Car le plus dur, c'est de faire passer le second au poste de premier."

Concernant la recherche d'un successeur en externe, cela implique de définir des critères et une méthode. Désormais, elle va s'orienter vers quelqu'un "qui a envie de relever le défi, qui voit [l'entreprise] et qui a envie de la porter. " Pour une Scop, la priorité, c'est " un très bon manager [...] capable de la développer dans un environnement concurrentiel qui sera le sien dans les dix ans". Au critère de compétence s'ajoutent cependant des critères humains: "On demande plus aux personnes en termes de développement personnel et d'investissement dans l'entreprise [...]. Etre associé, ce n'est pas négligeable, ce n'est pas neutre et ce n'est pas toujours 
facile de bien [le] faire comprendre. " Cet interlocuteur a ainsi finalement renoncé à envisager sa succession avec une personne recrutée préalablement pour diriger un nouveau site d'activité en filiale: "C'est un bon manager classique, mais je pense qu'il n'investira pas suffisamment sur un travail de management coopératif ", aspect sur lequel il reconnaît n'avoir pas assez insisté au moment du recrutement.

Par ailleurs, la méthode consiste à confier l'opération au PDG en place: "Je choisirai mon successeur [...] et on aura un plan de présentation aux associés. [...] Ce ne sont pas les associés actionnaires qui vont choisir le successeur... Je vais faire mon marché... Je suis en train de réfléchir avec deux cabinets... Et puis, je rencontre aujourd'hui deux personnes qui viennent par le biais d'un ami dirigeant d'une Scop. [...] C'est vrai, jaurais préféré rencontrer sur mon chemin l'homme providentiel. "Il semble ainsi que l'accent soit mis sur les affinités personnelles, la proximité idéologique et la séduction réciproque.

Enfin, la phase de règne conjoint, caractérisée par le transfert des connaissances, peut être organisée de manière originale. Dans un cas, le jeu se joue en deux étapes: le successeur choisi est recruté pour diriger le département le plus important durant deux ans, puis il entre au conseil d'administration de la coopérative avec une période de couverture déterminée d'avance (trois ans): "Je fais un autre mandat plein en termes de CA et [...] il sait quien 2008 il devient PDG de la société. "Dans un autre cas, il n'y a pas vraiment de double commande: la direction générale est confiée sans délai au successeur, "ce sera le patron opérationnel", tandis que le prédécesseur restera président du conseil d'administration et aura la responsabilité du développement externe. Le parcours commun est néanmoins présent: "Il y en a un qui découvre l'entreprise et l'autre qui la connaît parfaitement. [...] Il faut jouer l'empathie des deux côtés de façon à ne pas brutaliser ce passage de relais, notamment au regard des associés. " C'est donc la phase où le risque de tensions entre le prédécesseur et le successeur est le plus grand et la Scop n'est pas à l'abri d'un renversement de tendance où "la défiance succède à la confiance, l'opposition ouverte à la complicité ». Le doute s'installe sur la compétence du nouveau qui choisit de s'affirmer seul avec un transfert partiel de connaissances, sans appui bienveillant ni apport d'expériences du cédant. La gestion de la crise incombe alors au conseil d'administration des coopérateurs.

\section{Rôle des coopérateurs dans l'accueil du nouveau dirigeant}

Les salariés coopérateurs des Scop n'interviennent en général pas dans le choix du successeur, mais ils sont sollicités pour accepter ou invalider ce choix au moment où le successeur se présente pour l'élection au conseil d'administration avant de prendre ses fonctions de PDG. Il arrive ainsi qu'il soit recalé par manque de préparation de la part du prédécesseur et qu'il faille retarder son entrée officielle de quelques mois. De même, les membres du CA peuvent être amenés à gérer un conflit entre prédécesseur et successeur si le désengagement se passe mal et à choisir entre 
(22) Jusqu'à deux ou trois ans, contrairement aux entreprises classiques, où elle est réduite à la découverte de la solution à la succession en phase terminale. les deux leaders: "La mort dans l'âme les sociétaires durent voter. Le fondateur quitta l'entreprise fort amer " (Gouil, 2006, p. 2). Ainsi s'est exprimée la « sagesse » du collectif. L'intégration du nouveau dirigeant recruté en externe peut être facilitée en temps de crise. Les coopérateurs n'ont pas le choix, mais le redressement est vécu collectivement: "J'ai réussi, la société a réussi parce que ce n’est pas le travail d'un seul homme. "Plus généralement, durant la phase préparatoire du processus de succession, qui peut être assez longue ${ }^{(22)}$, les salariés-associés ont besoin d'être informés et rassurés pour bien appréhender la réalité de la situation et surmonter l'inquiétude liée au prochain changement de dirigeant.

Le témoignage des quatre personnes interrogées invite à un rapprochement entre les modalités de succession interne de PDG dans une Scop et les aspects humains de la transmission par un patron de sa PME à ses salariés, que ce soit à propos des étapes préparatoires au changement, des facilités de désengagement du prédécesseur, ou cédant, ou encore de la socialisation du nouveau dirigeant recruté en externe ou promu en interne. Les démarches évoquées pour assurer la succession dans les meilleures conditions sont autant de références qui servent à aborder les processus de transformation de PME classiques en coopératives. Cependant, la faisabilité de telles opérations n'est pas acquise en toutes circonstances.

\section{Les particularités de la transmission de PME en Scop}

L'enquête a permis d'identifier quelques éléments favorisants et de précaution, concernant autant la triple relation humaine entre le dirigeantcédant, le successeur-dirigeant élu et les salariés appelés au rachat de l'entreprise en devenant coopérateurs que le rôle des acteurs du mouvement Scop.

\section{Des facteurs favorisants}

En premier lieu, l'ouverture du mouvement Scop sur l'acceptation de dirigeants extérieurs depuis une quinzaine d'années a favorisé l'idée de la transmission de PME en Scop. On constate en effet que ces dirigeants de type "héliporté " possédant une expérience professionnelle dans les entreprises classiques ont un meilleur discours avec les cédants de PME, d'une part pour leur montrer leur propre réussite en Scop et les atouts du modèle, d'autre part pour les préparer à accepter de nouveaux questionnements sur la valeur de leur entreprise et notamment sur le potentiel humain représenté par leurs salariés. "Il ne faut pas arriver avec l'étendard Scop du jour au lendemain, il faut que ce soit un processus évolutif, [...] lorsquion arrive à aborder le sujet avec des cédants, si l'on a un peu d'empathie et que l'on sait écouter, ils vous relatent très rapidement leurs problèmes. "Il s'agit plus de semer et de laisser l'idée faire son chemin: "J'en connais une, je regarde, je vois un petit peu, on en a parlé... " Ainsi le cédant, qui a souvent une notoriété locale, est-il rassuré quand il perçoit que dans la coopérative le capital humain est plus important que le capital 
(23) Sans éluder la question financière, qui trouvera toujours sa solution.

(24) Au sein du comité d'entreprise, lieu privilégié des discussions, des échanges et de la négociation avec l'ensemble du personnel. financier: "Il peut sortir et aller le dimanche à la messe la tête haute." En deuxième lieu, selon les interlocuteurs interrogés, le processus de transformation doit répondre à certaines conditions qui constituent un potentiel favorable. Tout d'abord, il faut surtout convaincre le cédant très en amont que c'est effectivement la bonne solution pour pérenniser son entreprise selon ses vœux d'un "fonctionnement indépendant, responsable et autonome " ${ }^{(23)}$. L'étape suivante consiste à repérer quatre ou cinq personnes qui seraient aptes à dire: "Eh bien, oui, ça m'intéresse de continuer et d'avoir un autre rôle dans l'entreprise. "Il s'agit ainsi d'opérer une rupture psychologique et mentale sur le sujet, notamment au sein de la hiérarchie, et d'approcher les gens les plus concernés par le dispositif afin de constituer un noyau de départ. De plus, il paraît nécessaire que le management pratiqué par le cédant ait un caractère un tant soit peu participatif et transparent et que l'ambiance soit très peu conflictuelle, car il s'agit de mobiliser des salariés pour un nouveau projet qui va les impliquer ${ }^{(24)}$. Enfin, "il faut aller chercher un leader [...] capable de fédérer un groupe". En interne, on retrouve le problème de la légitimité, et en externe, celui de la distance, de la confiance à construire et de l'inquiétude sur la réussite de la "greffe ". Le cédant est confronté alors à une double démarche: la cession de son bien à ses salariés sous forme coopérative et la transmission de la direction à un nouveau dirigeant qu'il n'a pas forcément choisi et qui peut être inconnu des coopérateurs.

\section{La question de l'implication des salariés dans la transmission}

La difficulté réside dans la manière d'approcher les salariés en situation de transition pour les impliquer dans le processus de transformation de façon progressive: "Il ne faut pas les amener de but en blanc à prendre la casquette "associé-capital-gestion-responsabilité". [...] On ne change pas une culture, des comportements du jour au lendemain. "Une phase initiatique semble ainsi être nécessaire: "Vous allez avoir un nouveau dirigeant, la formule est un peu différente, on va vous faire participer davantage, on va vous aider financièrement. " Le réseau Scop est alors déterminant pour assurer cet accompagnement avec un discours évolutif sur les valeurs coopératives et un parrainage qui s'apparente à une forme d'adoption: il s'agit de s'appuyer sur le vécu des personnes pour leur faire appréhender leur nouveau rôle en tant quassociés et percevoir les avantages du statut. "Aller trop vite, passer du schéma de salarié à actionnaire, ça peut être néfaste à partir du moment où il n'y a pas de prise de conscience. " Le risque est tout simplement soit le rejet de la formule coopérative, soit des comportements erratiques aux assemblées générales: "Il faut un travail en amont, car on ne peut pas dire à des gens qui ont eu l'habitude d'être commandés, pilotés: vive la démocratie, c'est vous qui avez le pouvoir! "Enfin, s'il est difficile de transformer un nouveau salarié en coopérateur, il est encore plus spécifiquement délicat sur le plan de la gouvernance d'intégrer dans un processus de création d'une coopérative un groupe représentant une ou plusieurs dizaines de personnes d'un seul coup. 


\section{Conclusion}

Le changement de direction dans une PME étant d'abord un changement de personne, le facteur humain est donc déterminant dans la réussite ou l'échec du passage de relais. Le changement de dirigeant est aussi bien souvent l'occasion d'introduire un nouveau style de management et de nouvelles méthodes de travail. Les Scop sont également confrontées à ces moments singulièrement délicats et engageant leur avenir. Fondée sur un management démocratique où le conseil d'administration, composé des salariés élus, joue un rôle particulier, la Scop est une formule originale qui a permis entre autres choses de pérenniser des entreprises PME dans des conditions spécifiques. Et qu'il s'agisse d'une succession de dirigeant au sein d'une Scop ou d'une transformation de PME en Scop, l'intégration du nouveau comme le désengagement de l'ancien sont facilités par rapport aux cas d'entreprises familiales classiques. Le deuil est généralement plus aisé à faire en raison du non-transfert de propriété ou parce que l'on ne cède pas à un inconnu (les salariés). La bonne intégration du nouveau dirigeant est bien sûr liée à la confiance qu'il saura susciter, mais le rôle du conseil d'administration est là encore une source de socialisation et donc un facteur favorisant la réussite du processus, notamment au regard de la diffusion des valeurs coopératives. Les méthodes utilisées par les dirigeants de Scop pour organiser leur succession lors de leur départ à la retraite constituent un témoignage précieux dans ce travail exploratoire pour anticiper les problématiques spécifiques sur le plan humain d'une transformation d'entreprise classique saine en Scop. Dès lors, et compte tenu de l'ampleur du marché de la transmission dans les années à venir, notre recherche a permis d'identifier un élément de profil de PME à transformer en Scop, à savoir l'existence d'un management déjà participatif constituant pour la future coopérative un « ferment » à cultiver au cours d'un processus long et évolutif. Les dires et les points de vue des quelques acteurs-ressources, qui constituent l'intérêt de ce travail, mais aussi ses limites, offrent néanmoins des pistes plus précises pour l'étape suivante de la recherche, qui sera de procéder à des études de cas approfondies de transformation de PME saines en Scop. 


\section{Bibliographie}

Barbot M.-C., Richomme-Huet K., 2004, "Proposition d'un outil de pilotage pour la transmission des PME ", IVe colloque "Métamorphose des organisations ", université de Nancy 2, 21-22 octobre.

Bataille-Chédotel F., Huntzinger F., 2004, "L'entrepreneuriat collectif: modèle unique ou gouvernances multiples? Une approche exploratoire auprès de dix sociétés coopératives de production françaises ", en français dans Economie et Solidarités, Ciriec Canada, vol. 35, no 1-2.

Boussaguet S., 2003, «Le rôle des salariés dans le processus de socialisation du repreneur dans un contexte de PME ", in D. Retour, C. Defélix, M. Matmati (édit.), Actes $d u$ $X I V^{e}$ congrès $A G R H$, "GRH: innovons!", Grenoble, 20-22 novembre, tome I, p. 407-435. Cadieux L., 2005, «La succession dans les PME familiales: proposition d'un modèle de réussite du processus de désengagement du prédécesseur ", Revue internationale PME, Pressse de l'université du Québec, vol. 18, $n^{\circ} 3-4$, p. 31-49.

Fattoum S., Fayolle A., 2002, "L'impact de la relation prédécesseur-successeur sur le déroulement du processus de succession dans les entreprises familiales ", Actes du IVe congrès de l'Académie de l'entrepreneuriat, Paris.

Gueye Ch., Bah T., 2003, "La prise en compte de l'environnement psychologique et le transfert des connaissances dans le cadre d'une transmission d'entreprise familiale", in D. Retour, C. Defélix, M. Matmati (édit.),
Actes du XIVe congrès AGRH, "GRH: innovons! ", Grenoble, 20-22 novembre, tome II, pp. 1359-1376.

Gomez P.-Y., 1996, Le gouvernement de l'entreprise, Inter Editions, Paris.

Gouil H., 2006, «Transmettre sans céder », III $^{e}$ Carrefour universitaire du management Scop, « La gouvernance des Scop en question? Regards croisés ", Le Mans, université du Maine, 5 avril.

Le Breton-Miller I., Miller D., 2004, "Toward an integrative model of effective FOB succession ", Enterpreneuship Theory and Practice, été, p. 305-328.

Mahé de Boislandelle H., 1998, Gestion des ressources humaines dans les PME, Paris, Economica, $1^{\text {re édition } 1988 .}$

Pailot P., 1998, "Propositions théoriques et épistémologiques pour une méthodologie d'analyse des freins psychologiques des dirigeants de PME lors des transmissions d'entreprise ", CIFPME, IAE de Metz, octobre.

Reynaud J.-D., Richebé N., 2007, « Règles, conventions et valeurs, plaidoyer pour la normalité ordinaire ", Revue française de sociologie, vol. 48-1, p. 3-36.

Saint-Cyr L., Richer F., 2005, "La planification du processus de transmission dans les PME québécoises ", Revue internationale PME, Presses de l'université du Québec, vol. 18, $n^{\circ} 3-4$, p. 51-71.

Wacheux F., 1996, Méthodes qualitatives et recherche en gestion, Paris, Economica. 